\title{
The Determinants, Inverses, Norm, and Spread of Skew Circulant Type Matrices Involving Any Continuous Lucas Numbers
}

\author{
Jin-jiang Yao and Zhao-lin Jiang \\ Department of Mathematics, Linyi University, Linyi, Shandong 276000, China \\ Correspondence should be addressed to Zhao-lin Jiang; jzh1208@sina.com
}

Received 6 January 2014; Accepted 17 February 2014; Published 17 April 2014

Academic Editor: Feng Gao

Copyright ( 2014 J.-j. Yao and Z.-1. Jiang. This is an open access article distributed under the Creative Commons Attribution License, which permits unrestricted use, distribution, and reproduction in any medium, provided the original work is properly cited.

\begin{abstract}
We consider the skew circulant and skew left circulant matrices with any continuous Lucas numbers. Firstly, we discuss the invertibility of the skew circulant matrices and present the determinant and the inverse matrices by constructing the transformation matrices. Furthermore, the invertibility of the skew left circulant matrices is also discussed. We obtain the determinants and the inverse matrices of the skew left circulant matrices by utilizing the relationship between skew left circulant matrices and skew circulant matrix, respectively. Finally, the four kinds of norms and bounds for the spread of these matrices are given, respectively.
\end{abstract}

\section{Introduction}

Circulant and skew-circulant matrices are appearing increasingly often in scientific and engineering applications. Briefly, scanning the recent literature, one can see their utility is appreciated in the design of digital filters [1-3], image processing [4-6], communications [7], signal processing [8], and encoding [9]. They have been put on firm basis with the work of Davis [10] and Jiang and Zhou [11].

The skew circulant matrices as preconditioners for linear multistep formulae- (LMF-) based ordinary differential equations (ODEs) codes. Hermitian and skew-Hermitian Toeplitz systems are considered in [12-15]. Lyness and Sørevik employed a skew circulant matrix to construct $s$ dimensional lattice rules in [16]. Spectral decompositions of skew circulant and skew left circulant matrices were discussed in [17]. Compared with cyclic convolution algorithm, the skew cyclic convolution algorithm [8] is able to perform filtering procedure in approximate half of computational cost for real signals. In [2] two new normal-form realizations are presented which utilize circulant and skew circulant matrices as their state transition matrices. The well-known secondorder coupled form is a special case of the skew circulant form. Li et al. [18] gave the style spectral decomposition of skew circulant matrix firstly and then dealt with the optimal backward perturbation analysis for the linear system with skew circulant coefficient matrix. In [3], a new fast algorithm for optimal design of block digital filters (BDFs) was proposed based on skew circulant matrix.

Besides, some scholars have given various algorithms for the determinants and inverses of nonsingular circulant matrices $[10,11]$. Unfortunately, the computational complexity of these algorithms is very amazing with the order of matrix increasing. However, some authors gave the explicit determinants and inverse of circulant and skew circulant involving some famous numbers. For example, Jaiswal evaluated some determinants of circulant whose elements are the generalized Fibonacci numbers [19]. Lind presented the determinants of circulant and skew circulant involving the Fibonacci numbers [20]. Dazheng [21] gave the determinant of the Fibonacci-Lucas quasicyclic matrices. Shen et al. considered circulant matrices with the Fibonacci and Lucas numbers and presented their explicit determinants and inverses by constructing the transformation matrices [22]. Gao et al. [23] gave explicit determinants and inverses of skew circulant and skew left circulant matrices with the Fibonacci and Lucas numbers. Jiang et al. $[24,25]$ considered the skew circulant 
and skew left circulant matrices with the $k$-Fibonacci numbers and the $k$-Lucas numbers and discussed the invertibility of the these matrices and presented their determinant and the inverse matrix by constructing the transformation matrices, respectively.

Recently, there are several papers on the norms of some special matrices. Solak [26] established the lower and upper bounds for the spectral norms of circulant matrices with the classical Fibonacci and Lucas numbers entries. İpek [27] investigated an improved estimation for spectral norms of these matrices. Shen and Cen [28] gave upper and lower bounds for the spectral norms of $r$-circulant matrices in the forms of $A=C_{r}\left(F_{0}, F_{1}, \ldots, F_{n-1}\right), B=C_{r}\left(L_{0}, L_{1}, \ldots, L_{n-1}\right)$, and they also obtained some bounds for the spectral norms of Kronecker and Hadamard products of matrix $A$ and matrix $B$. Akbulak and Bozkurt [29] found upper and lower bounds for the spectral norms of Toeplitz matrices such that $a_{i j} \equiv$ $F_{i-j}$ and $b_{i j} \equiv L_{i-j}$. The convergence in probability and in distribution of the spectral norm of scaled Toeplitz, circulant, reverse circulant, symmetric circulant, and a class of $k$ circulant matrices is discussed in [30].

Beginning with Mirsky [31], several authors [32-38] have obtained bounds for the spread of a matrix.

The purpose of this paper is to obtain the explicit determinants, explicit inverses, norm, and spread of skew circulant type matrices involving any continuous Lucas numbers. And we generalize the result [23]. In passing, the norm and spread of skew circulant type matrices have not been researched. It is hoped that this paper will help in changing this. More work continuing the present paper is forthcoming.

In the following, let $r$ be a nonnegative integer. We adopt the following two conventions $0^{0}=1$, and, for any sequence $\left\{a_{n}\right\}, \sum_{k=i}^{n} a_{k}=0$ in the case $i>n$.

The Lucas sequences are defined by the following recurrence relations [21-23, 27-29]:

$$
L_{n+1}=L_{n}+L_{n-1}, \quad \text { where } L_{0}=2, L_{1}=1,
$$

for $n \geq 0$. The first few values of the sequences are given by the following table:

$$
\begin{array}{c|cccccccccc}
n & 0 & 1 & 2 & 3 & 4 & 5 & 6 & 7 & 8 & 9 \\
\hline L_{n} & 2 & 1 & 3 & 4 & 7 & 11 & 18 & 29 & 47 & 76
\end{array} .
$$

The $\left\{L_{n}\right\}$ is given by the formula

$$
L_{n}=\alpha^{n}+\beta^{n},
$$

where $\alpha$ and $\beta$ are the roots of the characteristic equation $x^{2}-$ $x-1=0$.

Definition 1 (see [17]). A skew circulant matrix over $C$ with the first row $\left(a_{1}, a_{2}, \ldots, a_{n}\right)$ is meant a square matrix of the form

$$
\left(\begin{array}{ccccc}
a_{1} & a_{2} & \ldots & a_{n-1} & a_{n} \\
-a_{n} & a_{1} & a_{2} & \ldots & a_{n-1} \\
\vdots & -a_{n} & a_{1} & \ddots & \vdots \\
-a_{3} & \vdots & \ddots & \ddots & a_{2} \\
-a_{2} & -a_{3} & \ldots & -a_{n} & a_{1}
\end{array}\right)_{n \times n},
$$

denoted by $\operatorname{SCirc}\left(a_{1}, a_{2}, \ldots, a_{n}\right)$.
Definition 2 (see [17]). A skew left circulant matrix over $C$ with the first row $\left(a_{1}, a_{2}, \ldots, a_{n}\right)$ is meant a square matrix of the form

$$
\left(\begin{array}{ccccc}
a_{1} & a_{2} & a_{3} & \cdots & a_{n} \\
a_{2} & a_{3} & \cdots & a_{n} & -a_{1} \\
a_{3} & \cdot & \cdot & . & \vdots \\
\vdots & a_{n} & -a_{1} & \cdots & -a_{n-2} \\
a_{n} & -a_{1} & \cdots & -a_{n-2} & -a_{n-1}
\end{array}\right)_{n \times n},
$$

denoted by $\operatorname{SLCirc}\left(a_{1}, a_{2}, \ldots, a_{n}\right)$.

Lemma 3 (see $[10,17])$. Let $A=\operatorname{SCirc}\left(a_{1}, a_{2}, \ldots, a_{n}\right)$ be skew circulant matrix; then

(i) $A$ is invertible if and only if the eigenvalues of $A$

$$
\lambda_{k}=f\left(\omega^{k} \eta\right) \neq 0, \quad(k=0,1,2, \ldots, n-1),
$$

where $f(x)=\sum_{j=1}^{n} a_{j} x^{j-1}, \omega=\exp (2 \pi i / n)$, and $\eta=$ $\exp (\pi i / n)$

(ii) if $A$ is invertible, then the inverse of $A$ is a skew circulant matrix.

Lemma 4 (see [17]). Let $A=\operatorname{SLCirc}\left(a_{1}, a_{2}, \ldots, a_{n}\right)$ be skew left circulant matrix and let $n$ be odd; then

$$
\begin{gathered}
\lambda_{j}= \pm\left|\sum_{k=1}^{n} a_{k} \omega^{(j-(1 / 2))(k-1)}\right|, \quad\left(j=1,2, \ldots, \frac{n-1}{2}\right), \\
\lambda_{(n+1) / 2}=\sum_{k=1}^{n}\left|a_{k}(-1)^{k-1}\right|,
\end{gathered}
$$

where $\lambda_{j}, j=1,2, \ldots,(n-1) / 2,(n+1) / 2$ are the eigenvalues of $A$.

Lemma 5 (see [23]). With the orthogonal skew left circulant matrix

$$
\Theta:=\left(\begin{array}{ccccc}
1 & 0 & \cdots & 0 & 0 \\
0 & 0 & \cdots & 0 & -1 \\
0 & 0 & \cdots & -1 & 0 \\
\vdots & \vdots & \ddots & \vdots & \vdots \\
0 & -1 & \cdots & 0 & 0
\end{array}\right)_{n \times n}
$$

it holds that

$$
\operatorname{SCirc}\left(a_{1}, a_{2}, \ldots, a_{n}\right)=\Theta \operatorname{SLCirc}\left(a_{1}, a_{2}, \ldots, a_{n}\right)
$$

Lemma 6 (see [23]). If

$$
\left[\operatorname{SCirc}\left(a_{1}, a_{2}, \ldots, a_{n}\right)\right]^{-1}=\operatorname{SCirc}\left(b_{1}, b_{2}, \ldots, b_{n}\right),
$$

then

$$
\left[\operatorname{SLCirc}\left(a_{1}, a_{2}, \ldots, a_{n}\right)\right]^{-1}=\operatorname{SLCirc}\left(b_{1},-b_{n}, \ldots,-b_{2}\right) .
$$


Lemma 7 (see $[27,28])$. Let $\left\{L_{n}\right\}$ be the Lucas numbers; then

$$
\begin{aligned}
& \text { (i) } \sum_{i=0}^{n-1} L_{i}=L_{n+1}-1 \\
& \text { (ii) } \sum_{i=0}^{n-1} L_{i}^{2}=L_{n} L_{n-1}+2 \\
& \text { (iii) } \sum_{i=0}^{n-1} i L_{i}=(n-1) L_{n+1}-L_{n+2}+4 .
\end{aligned}
$$

Definition 8 (see [29]). Let $A=\left(a_{i j}\right)$ be an $n \times n$ matrix. The maximum column sum matrix norm, the spectral norm, the Euclidean (or Frobenius) norm, and the maximum row sum matrix norm of matrix $A$ are, respectively,

$$
\begin{gathered}
\|A\|_{1}=\max _{1 \leq j \leq n} \sum_{i=1}^{n}\left|a_{i j}\right|, \\
\|A\|_{2}=\left(\max _{1 \leq i \leq n} \lambda_{i}\left(A^{*} A\right)\right)^{1 / 2}, \\
\|A\|_{F}=\left(\sum_{i, j=1}^{n}\left|a_{i j}\right|^{2}\right)^{1 / 2}, \\
\|A\|_{\infty}=\max _{1 \leq i \leq n} \sum_{j=1}^{n}\left|a_{i j}\right|,
\end{gathered}
$$

where $A^{*}$ denotes the conjugate transpose of $A$.

Lemma 9 (see [30]). If $A$ is an $n \times n$ real symmetric or normal matrix, then one has

$$
\|A\|_{2}=\max _{1 \leq i \leq n}\left|\lambda_{i}\right|,
$$

where $\lambda_{i}(i=1,2, \ldots, n)$ are the eigenvalues of $A$.

Definition 10 (see [31,32]). Let $A=\left(a_{i j}\right)$ be an $n \times n$ matrix with eigenvalues $\lambda_{i}, i=1,2, \ldots n$. The spread of $A$ is defined as

$$
s(A)=\max _{i, j}\left|\lambda_{i}-\lambda_{j}\right| .
$$

Beginning with Mirsky [31], several authors [32-38] have obtained bounds for the spread of a matrix.

Lemma 11. Let $A=\left(a_{i j}\right)$ be an $n \times n$ matrix. An upper bound for the spread due to Mirsky [31] states that

$$
s(A) \leqslant \sqrt{2\|A\|_{F}^{2}-\frac{2}{n}|\operatorname{tr} A|^{2}},
$$

where $\|A\|_{F}$ denotes the Frobenius norm of $A$ and $\operatorname{tr} A$ is trace of $A$.

Lemma 12 (see [38]). Let $A=\left(a_{i j}\right)$ be an $n \times n$ matrix; then

(i) if $A$ is real and normal, then

$$
s(A) \geq \frac{1}{n-1}\left|\sum_{i \neq j} a_{i j}\right|,
$$

(ii) and if $A$ is Hermitian, then

$$
s(A) \geq 2 \max _{i \neq j}\left|a_{i j}\right| .
$$

\section{Determinant and Inverse of Skew Circulant Matrix with the Lucas Numbers}

In this section, let $A_{r, n}=\operatorname{SCirc}\left(L_{r+1}, \ldots, L_{r+n}\right)$ be skew circulant matrix. Firstly, we give a determinant explicit formula for the matrix $A_{r, n}$. Afterwards, we prove that $A_{r, n}$ is an invertible matrix for $n \geq 2$, and then we find the inverse of the matrix $A_{r, n}$.

In the following, let $x=-\left(\left(L_{r}+L_{r+n}\right) /\left(L_{r+1}+L_{r+n+1}\right)\right), t=$ $L_{r+2} / L_{r+1}, c=L_{r+1}+L_{r+n+1}, d=L_{r}+L_{r+n}, l_{n}=L_{r+1}+t L_{r+n}+$ $\sum_{k=1}^{n-2}\left(t L_{r+k+1}-L_{r+k+2}\right) \cdot x^{n-(k+1)}$ and $l_{n}^{\prime}=\sum_{k=1}^{n-1} L_{r+k+1} \cdot x^{n-(k+1)}$.

Theorem 13. Let $A_{r, n}=\operatorname{SCirc}\left(L_{r+1}, \ldots, L_{r+n}\right)$ be skew circulant matrix; then

$$
\begin{aligned}
\operatorname{det} A_{r, n}=L_{r+1}[ & L_{r+1}+t L_{r+n} \\
& \left.+\sum_{i=1}^{n-2}\left(t L_{r+i+1}-L_{r+i+2}\right) x^{n-(i+1)}\right] \cdot c^{n-2},
\end{aligned}
$$

where $L_{r+n}$ is the $(r+n)$ th Lucas number. Specially, when $r=0$, one gets the result of [23].

Proof. Obviously, det $A_{r, 1}=L_{r+1}$ satisfies the equation. In the case $n>1$, let

$$
\begin{aligned}
& \Sigma=\left(\begin{array}{cccccccc}
1 & & & & & & \\
t & & & & & & 1 \\
1 & & & & & 1 & -1 \\
0 & & & 0 & & 1 & -1 & -1 \\
\vdots & & & & & & \\
0 & & & 1 & \ddots & \ddots & . & \\
0 & & 1 & -1 & \therefore & & & \\
0 & 1 & -1 & -1 & & & &
\end{array}\right) \\
& \Omega_{1}=\left(\begin{array}{cccccc}
1 & 0 & 0 & \cdots & 0 & 0 \\
0 & x^{n-2} & 0 & \cdots & 0 & 1 \\
0 & x^{n-3} & 0 & \cdots & 1 & 0 \\
\vdots & \vdots & \vdots & \ddots & \vdots & \vdots \\
0 & x & 1 & \cdots & 0 & 0 \\
0 & 1 & 0 & \cdots & 0 & 0
\end{array}\right)
\end{aligned}
$$

be two $n \times n$ matrices; then we have

$$
\Sigma A_{r, n} \Omega_{1}=\left(\begin{array}{cccccc}
L_{r+1} & l_{n}^{\prime} & c_{13} & \cdots & c_{1, n-1} & c_{1 n} \\
0 & l_{n} & c_{23} & \cdots & c_{2, n-1} & c_{2 n} \\
0 & 0 & c & & & \\
0 & 0 & d & \ddots & & \\
\vdots & \vdots & & \ddots & c & \\
0 & 0 & & & d & c
\end{array}\right),
$$


where

$$
\begin{array}{r}
c_{1 j}=L_{r+n+2-j}, \quad c_{2 j}=t L_{r+n+2-j}-L_{r+n+3-j}, \\
(j=3,4, \ldots, n) .
\end{array}
$$

So it holds that

$$
\begin{aligned}
& \operatorname{det} \Sigma \operatorname{det} A_{r, n} \operatorname{det} \Omega_{1} \\
& =L_{r+1}\left[L_{r+1}+t L_{r+n}\right. \\
& \left.\quad+\sum_{k=1}^{n-2}\left(t L_{r+k+1}-L_{r+k+2}\right) x^{n-(i+1)}\right] \\
& \quad \cdot\left(L_{r+1}+L_{r+n+1}\right)^{n-2} .
\end{aligned}
$$

While taking $\operatorname{det} \Sigma=\operatorname{det} \Omega_{1}=(-1)^{(n-1)(n-2) / 2}$, we have

$$
\begin{aligned}
& \operatorname{det} A_{r, n} \\
& =L_{r+1}\left[L_{r+1}+t L_{r+n}\right. \\
& \left.+\sum_{k=1}^{n-2}\left(t L_{r+k+1}-L_{r+k+2}\right) x^{n-(i+1)}\right] \\
& \cdot\left(L_{r+1}+L_{r+n+1}\right)^{n-2} .
\end{aligned}
$$

This completes the proof.

Theorem 14. Let $A_{r, n}=\operatorname{SCirc}\left(L_{r+1}, \ldots, L_{r+n}\right)$ be skew circulant matrix; then $A_{r, n}$ is an invertible matrix. Specially, when $r=0$, one gets the result of [23].

Proof. Taking $n=2$ in, Theorem 13, we have $\operatorname{det} A_{r, 2}=L_{r+1}^{2}+$ $L_{r+2}^{2} \neq 0$. Hence $A_{r, 2}$ is invertible. In the case $n>2$, since $L_{r+n}=\alpha^{r+n}+\beta^{r+n}$, where $\alpha+\beta=1, \alpha \beta=-1$, we have

$$
\begin{aligned}
f\left(\omega^{k} \eta\right) & =\sum_{j=1}^{n} L_{r+j}\left(\omega^{k} \eta\right)^{j-1} \\
& =\sum_{j=1}^{n}\left(\alpha^{r+j}+\beta^{r+j}\right)\left(\omega^{k} \eta\right)^{j-1} \\
& =\frac{\alpha^{r+1}\left(1+\alpha^{n}\right)}{1-\alpha \omega^{k} \eta}+\frac{\beta^{r+1}\left(1+\beta^{n}\right)}{1-\beta \omega^{k} \eta} \\
& =\frac{L_{r+1}+L_{r+n+1}+\left(L_{r}+L_{r+n}\right) \omega^{k} \eta}{1-\omega^{k} \eta-\omega^{2 k} \eta^{2}} \\
& (k=1,2, \ldots, n-1),
\end{aligned}
$$

where $\omega=\exp (2 \pi i / n), \eta=\exp (\pi i / n)$. If there exists $\omega^{l} \eta(l=$ $1,2, \ldots, n-1)$ such that $f\left(\omega^{l} \eta\right)=0$, we obtain $L_{r+1}+L_{r+n+1}+$ $\left(L_{r}+L_{r+n}\right) \omega^{l} \eta=0$, for $1-\omega^{l} \eta-\omega^{2 l} \eta^{2} \neq 0$, and hence it follows that $\omega^{l} \eta=-\left(\left(L_{r+1}+L_{r+n+1}\right) /\left(L_{r}+L_{r+n}\right)\right)$ is a real number. Since

$$
\begin{aligned}
\omega^{l} \eta & =\exp \frac{(2 l+1) \pi i}{n} \\
& =\cos \frac{(2 l+1) \pi}{n}+i \sin \frac{(2 l+1) \pi}{n},
\end{aligned}
$$

it yields that $\sin ((2 l+1) \pi / n)=0$, so we have $\omega^{l} \eta=-1$ for $0<(2 l+1) \pi / n<2 \pi$. Since $x=-1$ is not the root of the equation $L_{r+1}+L_{r+n+1}+\left(L_{r}+L_{r+n}\right) x=0(n>2)$. We obtain $f\left(\omega^{k} \eta\right) \neq 0$, for any $\omega^{k} \eta(k=1,2, \ldots, n-1)$, while

$$
\begin{aligned}
f(\eta) & =\sum_{j=1}^{n} L_{j} \eta^{j-1} \\
& =\frac{L_{r+1}+L_{r+n+1}+\left(L_{r}+L_{r+n}\right) \eta}{1-\eta-\eta^{2}} \neq 0 .
\end{aligned}
$$

It follows from Lemma 3 that the conclusion holds.

Lemma 15. Let the matrix $\mathscr{H}=\left[h_{i j}\right]_{i, j=1}^{n-2}$ be of the form

$$
h_{i j}= \begin{cases}L_{r+1}+L_{r+n+1}=c, & i=j, \\ L_{r}+L_{r+n}=d, & i=j+1, \\ 0, & \text { otherwise. }\end{cases}
$$

Then the inverse $\mathscr{H}^{-1}=\left[h_{i, j}^{\prime}\right]_{i, j=1}^{n-2}$ of the matrix $\mathscr{H}$ is equal to

$$
h_{i j}^{\prime}= \begin{cases}\frac{(-d)^{i-j}}{c^{i-j+1}}, & i \geq j, \\ 0, & i<j .\end{cases}
$$

Specially, when $r=0$, one gets the result of [23].

Proof. Let $e_{i j}=\sum_{k=1}^{n-2} h_{i k} h_{k j}^{\prime}$. Obviously, $e_{i j}=0$ for $i<j$. In the case $i=j$, we obtain $e_{i i}=h_{i i} h_{i i}^{\prime}=\left(L_{r+1}+L_{r+n+1}\right) \cdot\left(1 /\left(L_{r+1}+\right.\right.$ $\left.\left.L_{r+n+1}\right)\right)=1$. For $i \geq j+1$, we obtain

$$
\begin{aligned}
e_{i j} & =\sum_{k=1}^{n-2} h_{i k} h_{k j}^{\prime}=h_{i, i-1} h_{i-1, j}^{\prime}+h_{i i} h_{i j}^{\prime} \\
& =d \cdot \frac{(-d)^{i-j-1}}{c^{i-j}}+c \cdot \frac{(-d)^{i-j}}{c^{i-j+1}}=0 .
\end{aligned}
$$

Hence, we get $\mathscr{H} \mathscr{H}^{-1}=I_{n-2}$, where $I_{n-2}$ is $(n-2) \times(n-2)$ identity matrix. Similarly, we can verify that $\mathscr{H}^{-1} \mathscr{H}=I_{n-2}$. Thus, the proof is completed.

Theorem 16. Let $A_{r, n}=\operatorname{SCirc}\left(L_{r+1}, \ldots, L_{r+n}\right)$ be skew circulant matrix; then

$$
\left(A_{r, n}\right)^{-1}=\frac{1}{l_{n}} \cdot \operatorname{SCirc}\left(y_{1}^{\prime}, y_{2}^{\prime}, \ldots, y_{n}^{\prime}\right),
$$


where

$$
\begin{aligned}
& y_{1}^{\prime}=1- {\left[\left(L_{r+3}-t L_{r+2}\right) \cdot \frac{(-d)^{n-3}}{c^{n-2}}\right.} \\
&\left.+\sum_{i=1}^{n-3}\left(L_{r+n+2-i}-t L_{r+n+1-i}\right) \cdot \frac{(-d)^{i-1}}{c^{i}}\right], \\
& y_{2}^{\prime}=-t-\sum_{i=1}^{n-2}\left(L_{r+n+1-i}-t L_{r+n-i}\right) \cdot \frac{(-d)^{i-1}}{c^{i}}, \\
& y_{3}^{\prime}=-\left(L_{r+3}-t L_{r+2}\right) \cdot \frac{1}{c} \\
& y_{4}^{\prime}=-\sum_{i=1}^{2}\left(L_{r+1+i}-t L_{r+i}\right) \cdot \frac{(-d)^{i-1}}{c^{i}} \\
& y_{k}^{\prime}=-\sum_{i=1}^{2}\left(L_{r+1+i}-t L_{r+i}\right) \cdot \frac{(-d)^{k-5+i}}{c^{k-4+i}} \\
& \quad(k=5,6, \ldots, n) .
\end{aligned}
$$

Specially, when $r=0$, one gets the result of [23].

Proof. Let

$$
\Omega_{2}=\left(\begin{array}{cccccc}
1 & -\frac{l_{n}^{\prime}}{L_{r+1}} & \omega_{13} & \omega_{14} & \cdots & \omega_{1 n} \\
0 & 1 & \omega_{23} & \omega_{24} & \cdots & \omega_{2 n} \\
0 & 0 & 1 & 0 & \cdots & 0 \\
0 & 0 & 0 & 1 & \cdots & 0 \\
\vdots & \vdots & \vdots & \vdots & \ddots & \vdots \\
0 & 0 & 0 & 0 & \cdots & 1
\end{array}\right),
$$

where

$$
\begin{gathered}
\omega_{1 j}=\frac{1}{L_{r+1}}\left[\frac{l_{n}^{\prime}}{l_{n}}\left(t L_{r+n+2-j}-L_{r+n+3-j}\right)-L_{r+n+2-j}\right] \\
\omega_{2 j}=\frac{1}{l_{n}} \cdot\left(L_{r+n+3-j}-t L_{r+n+2-j}\right) \quad(j=3,4, \ldots, n) .
\end{gathered}
$$

Then, we have

$$
\Sigma A_{r, n} \Omega_{1} \Omega_{2}=\left(\begin{array}{cccccc}
L_{r+1} & 0 & 0 & 0 & \cdots & 0 \\
0 & l_{n} & 0 & 0 & \cdots & 0 \\
0 & 0 & c & 0 & \cdots & 0 \\
0 & 0 & d & c & \cdots & 0 \\
\vdots & \vdots & \vdots & \vdots & \ddots & \vdots \\
0 & 0 & 0 & 0 & \cdots & c
\end{array}\right)
$$

so $\Sigma A_{r, n} \Omega_{1} \Omega_{2}=\mathscr{D} \oplus \mathscr{H}$, where $D=\operatorname{diag}\left(L_{r+1}, l_{n}\right)$ is a diagonal matrix and $\mathscr{D} \oplus \mathscr{H}$ is the direct sum of $\mathscr{D}$ and $\mathscr{H}$. If we denote $\Omega=\Omega_{1} \Omega_{2}$, then we obtain $A_{r, n}^{-1}=\Omega\left(\mathscr{D}^{-1} \oplus \mathscr{H}^{-1}\right) \Sigma$.
Since the last row elements of the matrix $\Omega$ are $\left(0,1, \omega_{23}, \omega_{24}, \ldots, \omega_{2, n-1}, \omega_{2 n}\right)$, then the last row elements of the matrix $\Omega\left(\mathscr{D}^{-1} \oplus \mathscr{H}^{-1}\right)$ are $\left(0,1 / l_{n}, T_{23}, T_{24}, \ldots, T_{2 n}\right)$, where

$$
\begin{gathered}
T_{23}=\sum_{i=1}^{n-2} \omega_{2,2+i} \cdot \frac{(-d)^{i-1}}{c^{i}}, \\
T_{2 k}=\sum_{i=1}^{n+1-k} \omega_{2, k-1+i} \cdot \frac{(-d)^{i-1}}{c^{i}} \quad(k=3,4, \ldots, n) .
\end{gathered}
$$

Hence, it follows from Lemma 15 that letting $A_{r, n}^{-1}=$ $\operatorname{SCirc}\left(y_{1}, y_{2}, \ldots, y_{n}\right)$, then its last row elements are $\left(-y_{2}\right.$, $\left.-y_{3}, \ldots,-y_{n}, y_{1}\right)$ which are given by the following equations:

$$
\begin{aligned}
-y_{2} & =\frac{t}{l_{n}}+T_{23} \\
& =\frac{t}{l_{n}}+\frac{1}{l_{n}} \sum_{i=1}^{n-2}\left(L_{r+n+1-i}-t L_{r+n-i}\right) \cdot \\
-y_{3} & =T_{2, n}=\frac{1}{l_{n}}\left(L_{r+3}-t L_{r+2}\right) \cdot \frac{1}{c}, \\
-y_{4} & =T_{2, n-1}-T_{2 n} \\
& =\frac{1}{l_{n}} \sum_{i=1}^{2}\left(L_{r+1+i}-t L_{r+i}\right) \cdot \frac{(-d)^{i-1}}{c^{i}}, \\
-y_{5} & =T_{2, n-2}-T_{2 n-1}-T_{2 n} \\
& =\frac{1}{l_{n}} \sum_{i=1}^{2}\left(L_{r+1+i}-t L_{r+i}\right) \cdot \frac{(-d)^{i}}{c^{i+1}},
\end{aligned}
$$$$
=\frac{t}{l_{n}}+\frac{1}{l_{n}} \sum_{i=1}^{n-2}\left(L_{r+n+1-i}-t L_{r+n-i}\right) \cdot \frac{(-d)^{i-1}}{c^{i}},
$$

$$
\begin{aligned}
-y_{k} & =T_{2, n-k+3}-T_{2, n-k+4}-T_{2, n-k+5} \\
& =\frac{1}{l_{n}} \sum_{i=1}^{2}\left(L_{r+1+i}-t L_{r+i}\right) \cdot \frac{(-d)^{k-5+i}}{c^{k-4+i}},
\end{aligned}
$$

$$
\begin{aligned}
-y_{n}= & T_{23}-T_{24}-T_{25} \\
= & \sum_{i=1}^{n-2} \omega_{2,2+i} \cdot \frac{(-d)^{i-1}}{c^{i}}-\sum_{i=1}^{n-3} \omega_{2,3+i} \cdot \frac{(-d)^{i-1}}{c^{i}} \\
& -\sum_{i=1}^{n-4} \omega_{2,4+i} \cdot \frac{(-d)^{i-1}}{c^{i}} \\
= & \frac{1}{l_{n}} \sum_{i=1}^{2}\left(L_{r+1+i}-t L_{r+i}\right) \cdot \frac{(-d)^{n-5+i}}{c^{n-4+i}},
\end{aligned}
$$




$$
\begin{aligned}
y_{1}=\frac{1}{l_{n}}-T_{23} & -T_{24} \\
=\frac{1}{l_{n}}-\frac{1}{l_{n}}[ & \left(L_{r+3}-t L_{r+2}\right) \cdot \frac{(-d)^{n-3}}{c^{n-2}} \\
& \left.+\sum_{i=1}^{n-3}\left(L_{r+n+2-i}-t L_{r+n+1-i}\right) \cdot \frac{(-d)^{i-1}}{c^{i}}\right] .
\end{aligned}
$$

Hence, we obtain

$$
\begin{aligned}
& y_{1}= \frac{1}{l_{n}}-\frac{1}{l_{n}}\left[\left(L_{r+3}-t L_{r+2}\right) \cdot \frac{(-d)^{n-3}}{c^{n-2}}\right. \\
&\left.\quad+\sum_{i=1}^{n-3}\left(L_{r+n+2-i}-t L_{r+n+1-i}\right) \frac{(-d)^{i-1}}{c^{i}}\right], \\
& y_{2}=-\frac{t}{l_{n}}-\frac{1}{l_{n}} \sum_{i=1}^{n-2}\left(L_{r+n+1-i}-t L_{r+n-i}\right) \cdot \frac{(-d)^{i-1}}{c^{i}}, \\
& y_{3}=-\frac{1}{l_{n}}\left(L_{r+3}-t L_{r+2}\right) \cdot \frac{1}{c}, \\
& y_{4}=-\frac{1}{l_{n}} \sum_{i=1}^{2}\left(L_{r+1+i}-t L_{r+i}\right) \cdot \frac{(-d)^{i-1}}{c^{i}}, \\
& y_{5}=-\frac{1}{l_{n}} \sum_{i=1}^{2}\left(L_{r+1+i}-t L_{r+i}\right) \cdot \frac{(-d)^{i}}{c^{i+1}} \\
& y_{n}=\frac{1}{l_{n}} \sum_{i=1}^{2}\left(L_{r+1+i}-t L_{r+i}\right) \cdot \frac{(-d)^{n-5+i}}{c^{n-4+i}}, \\
& y_{k}=-\frac{1}{l_{n}} \sum_{i=1}^{2}\left(L_{r+1+i}-t L_{r+i}\right) \cdot \frac{(-d)^{k-5+i}}{c^{k-4+i}}, \\
& \vdots
\end{aligned}
$$

where

$$
\begin{aligned}
& y_{1}^{\prime}=1- {\left[\left(L_{r+3}-t L_{r+2}\right) \cdot \frac{(-d)^{n-3}}{c^{n-2}}\right.} \\
&\left.+\sum_{i=1}^{n-3}\left(L_{r+n+2-i}-t L_{r+n+1-i}\right) \cdot \frac{(-d)^{i-1}}{c^{i}}\right], \\
& y_{2}^{\prime}=-t-\sum_{i=1}^{n-2}\left(L_{r+n+1-i}-t L_{r+n-i}\right) \cdot \frac{(-d)^{i-1}}{c^{i}}, \\
& y_{3}^{\prime}=-\left(L_{r+3}-t L_{r+2}\right) \cdot \frac{1}{c}
\end{aligned}
$$

$$
\begin{gathered}
y_{4}^{\prime}=-\sum_{i=1}^{2}\left(L_{r+1+i}-t L_{r+i}\right) \cdot \frac{(-d)^{i-1}}{c^{i}}, \\
y_{k}^{\prime}=-\sum_{i=1}^{2}\left(L_{r+1+i}-t L_{r+i}\right) \cdot \frac{(-d)^{k-5+i}}{c^{k-4+i}}, \quad(k=5,6, \ldots, n) .
\end{gathered}
$$

This completes the proof.

\section{Norm and Spread of Skew Circulant Matrix with the Lucas Numbers}

Theorem 17. Let $A_{r, n}=\operatorname{SCirc}\left(L_{r+1}, \ldots, L_{r+n}\right)$ be skew circulant matrix; then three kinds of norms of $A_{r, n}$ are given by

$$
\begin{gathered}
\left\|A_{r, n}\right\|_{1}=\left\|A_{r, n}\right\|_{\infty}=L_{r+n+2}-L_{r+2} \\
\left\|A_{r, n}\right\|_{F}=\sqrt{n\left(L_{r+n} L_{r+n+1}-L_{r} L_{r+1}\right)} .
\end{gathered}
$$

Proof. By Definition 8 and (12), we have

$$
\left\|A_{r, n}\right\|_{1}=\left\|A_{r, n}\right\|_{\infty}=\sum_{i=1}^{n} L_{r+i}=L_{r+n+2}-L_{r+2} .
$$

By Definition 8 and (13), we have

$$
\begin{aligned}
\left(\left\|A_{r, n}\right\|_{F}\right)^{2} & =\sum_{i=1}^{n} \sum_{j=1}^{n}\left|a_{i j}\right|^{2} \\
& =n \sum_{i=1}^{n} L_{r+i}^{2} \\
& =n\left(\sum_{i=0}^{r+n} L_{i}^{2}-\sum_{i=0}^{r} L_{i}^{2}\right) \\
& =n\left(L_{r+n} L_{r+n+1}-L_{r} L_{r+1}\right) .
\end{aligned}
$$

Thus

$$
\left\|A_{r, n}\right\|_{F}=\sqrt{n\left(L_{r+n} L_{r+n+1}-L_{r} L_{r+1}\right)}
$$

Theorem 18. Let

$$
A_{r, n}^{\prime}=\operatorname{SCirc}\left(L_{r+1},-L_{r+2}, \ldots,-L_{r+n-1}, L_{r+n}\right)
$$

be an odd-order alternative skew circulant matrix and let $n$ be odd. Then

$$
\left\|A_{r, n}^{\prime}\right\|_{2}=\sum_{i=1}^{n} L_{r+i}=L_{r+n+2}-L_{r+2}
$$

Proof. By Lemma 3, we have

$$
\lambda_{j}\left(A_{r, n}^{\prime}\right)=\sum_{i=1}^{n}(-1)^{i-1} L_{r+i}\left(\omega^{j} \eta\right)^{i-1}
$$


So

$$
\begin{aligned}
\left|\lambda_{j}\left(A_{r, n}^{\prime}\right)\right| & \leq \sum_{i=1}^{n}\left|(-1)^{i-1} L_{r+i}\right| \cdot\left|\left(\omega^{j} \eta\right)^{i-1}\right| \\
& =\sum_{i=1}^{n} L_{r+i},
\end{aligned}
$$

for all $j=0,1, \ldots, n-1$.

Since $n$ is odd, $\sum_{i=1}^{n} L_{r+i}$ is an eigenvalue of $A_{r, n}^{\prime}$; that is,

$$
\begin{gathered}
\left(\begin{array}{cccc}
L_{r+1} & -L_{r+2} & \vdots & L_{r+n} \\
-L_{r+n} & L_{r+1} & -L_{r+n-1} \\
L_{r+n-1} & -L_{r+n} & L_{r+n-2} \\
\vdots & \vdots & \vdots & \vdots \\
L_{r+2} & -L_{r+3} & L_{r+1}
\end{array}\right)\left(\begin{array}{c}
1 \\
-1 \\
1 \\
-1 \\
\vdots \\
1
\end{array}\right) \\
=\sum_{i=1}^{n} L_{r+i} \cdot\left(\begin{array}{c}
1 \\
-1 \\
1 \\
-1 \\
\vdots \\
1
\end{array}\right) .
\end{gathered}
$$

To sum up, we have

$$
\max _{0 \leq j \leq n-1}\left|\lambda_{j}\left(A_{r, n}^{\prime}\right)\right|=\sum_{i=1}^{n} L_{r+i} .
$$

Since all skew circulant matrices are normal, by Lemma 9 and (12), and (52), we have

$$
\left\|A_{r, n}^{\prime}\right\|_{2}=\sum_{i=1}^{n} L_{r+i}=L_{r+n+2}-L_{r+2},
$$

which completes the proof.

Theorem 19. Let $A_{r, n}=\operatorname{SCirc}\left(L_{r+1}, \ldots, L_{r+n}\right)$ be skew circulant matrix; then the bounds for the spread of $A_{r, n}$ are

$$
\begin{gathered}
s\left(A_{r, n}\right) \leqslant \sqrt{2 n\left(L_{r+n} L_{r+n+1}-L_{r+1} L_{r+2}\right)}, \\
s\left(A_{r, n}\right) \geq \frac{1}{n-1}\left|2 L_{r+n+3}-(n-2) L_{r+n+2}-n L_{r+3}-2 L_{r+4}\right| .
\end{gathered}
$$

Proof. The trace of $A_{r, n}, \operatorname{tr} A_{r, n}=n L_{r+1}$. By (18) and (43), we have

$$
s\left(A_{r, n}\right) \leqslant \sqrt{2 n\left(L_{r+n} L_{r+n+1}-L_{r+1} L_{r+2}\right)} .
$$

Since

$$
\begin{aligned}
\sum_{i \neq j} a_{i j} & =\sum_{k=2}^{n}(n-(k-1)) L_{r+k}-\sum_{k=2}^{n}(k-1) L_{r+k} \\
& =(n+2) \sum_{k=2}^{n} L_{r+k}-2 \sum_{k=2}^{n} k L_{r+k}
\end{aligned}
$$

$$
\begin{aligned}
= & (n+2)\left(L_{r+n+2}-L_{r+3}\right) \\
& -2\left[\sum_{k=2}^{n}(r+k) L_{r+k}-\sum_{k=2}^{n} r L_{r+k}\right],
\end{aligned}
$$

by (12) and (14),

$$
\sum_{i \neq j} a_{i j}=2 L_{r+n+3}-(n-2) L_{r+n+2}-n L_{r+3}-2 L_{r+4} .
$$

By (19), we have

$$
\begin{gathered}
s\left(A_{r, n}\right) \geq \frac{1}{n-1} \mid 2 L_{r+n+3}-(n-2) L_{r+n+2} \\
-n L_{r+3}-2 L_{r+4} \mid .
\end{gathered}
$$

\section{Determinant and Inverse of Skew Left Circulant Matrix with the Lucas Numbers}

In this section, let $A_{r, n}^{\prime \prime}=\operatorname{SLCirc}\left(L_{r+1}, \ldots, L_{r+n}\right)$ be skew left circulant matrix. By using the obtained conclusions in Section 2, we give a determinant explicit formula for the matrix $A_{r, n}^{\prime \prime}$. Afterwards, we prove that $A_{r, n}^{\prime \prime}$ is an invertible matrix for any positive interger $n$. The inverse of the matrix $A_{r, n}^{\prime \prime}$ is also presented.

According to Lemmas 5 and 6 and Theorems 13, 14, and 16 , we can obtain the following theorems.

Theorem 20. Let $A_{r, n}^{\prime \prime}=\operatorname{SLCirc}\left(L_{r+1}, \ldots, L_{r+n}\right)$ be skew left circulant matrix; then

$$
\begin{aligned}
\operatorname{det} A_{r, n}^{\prime \prime}= & (-1)^{n(n-1) / 2} L_{r+1} \\
& \times\left[L_{r+1}+t L_{r+n}+\sum_{k=1}^{n-2}\left(t L_{r+1+i}-L_{r+2+i}\right) x^{n-1-i}\right] \\
& \cdot c^{n-2},
\end{aligned}
$$

where $L_{r+n}$ is the $(r+n)$ th Lucas number.

Theorem 21. Let $A_{r, n}^{\prime \prime}=\operatorname{SLCirc}\left(L_{r+1}, \ldots, L_{r+n}\right)$ be skew left circulant matrix; then $A_{r, n}^{\prime \prime}$ is an invertible matrix.

Theorem 22. Let $A_{r, n}^{\prime \prime}=\operatorname{SLCirc}\left(L_{r+1}, \ldots, L_{r+n}\right)$ be skew left circulant matrix; then

$$
\left(A_{r, n}^{\prime \prime}\right)^{-1}=\frac{1}{l_{n}} \operatorname{SLCirc}\left(y_{1}^{\prime \prime}, y_{2}^{\prime \prime}, \ldots, y_{n}^{\prime \prime}\right)
$$

where

$$
\begin{aligned}
y_{1}^{\prime \prime}=1- & {\left[\left(L_{r+3}-t L_{r+2}\right) \frac{(-d)^{n-3}}{c^{n-2}}\right.} \\
& \left.+\sum_{i=1}^{n-3}\left(L_{r+n+2-i}-t L_{r+n+1-i}\right) \cdot \frac{(-d)^{i-1}}{c^{i}}\right],
\end{aligned}
$$




$$
\begin{gathered}
y_{k}^{\prime \prime}=-y_{n-k+2}^{\prime} \\
=\sum_{i=1}^{2}\left(L_{r+1+i}-t L_{r+i}\right) \cdot \frac{(-d)^{n-k-3+i}}{c^{n-k-2+i}}, \\
\quad(k=2,3, \ldots, n-2) . \\
y_{n-1}^{\prime \prime}=-y_{3}^{\prime}=\left(L_{r+3}-t L_{r+2}\right) \cdot \frac{1}{c}, \\
y_{n}^{\prime \prime}=-y_{2}^{\prime} \quad t+\sum_{i=1}^{n-2}\left(L_{r+n+1-i}-t L_{r+n-i}\right) \cdot \frac{(-d)^{i-1}}{c^{i}} .
\end{gathered}
$$

\section{Norm and Spread of Skew Left Circulant Matrix with the Lucas Numbers}

Theorem 23. Let $A_{r, n}^{\prime \prime}=\operatorname{SLCirc}\left(L_{r+1}, \ldots, L_{r+n}\right)$ be skew left circulant matrix. Then three kinds of norms of $A_{r, n}^{\prime \prime}$ are given by

$$
\begin{gathered}
\left\|A_{r, n}^{\prime \prime}\right\|_{1}=\left\|A_{r, n}\right\|_{\infty}=L_{r+n+2}-L_{r+2}, \\
\left\|A_{r, n}^{\prime \prime}\right\|_{F}=\sqrt{n\left(L_{r+n} L_{r+n+1}-L_{r} L_{r+1}\right) .}
\end{gathered}
$$

Proof. Using the method in Theorem 17 similarly, the conclusion is obtained.

Theorem 24. Let

$$
A_{r, n}^{\prime \prime \prime}=\operatorname{SLCirc}\left(L_{r+1},-L_{r+2}, \ldots,-L_{r+n-1}, L_{r+n}\right)
$$

be an odd-order alternative skew left circulant matrix; then

$$
\left\|A_{r, n}^{\prime \prime \prime}\right\|_{2}=\sum_{i=1}^{n} L_{r+i}=L_{r+n+2}-L_{r+2} .
$$

Proof. According to Lemma 4,

$$
\lambda_{j}\left(A_{r, n}^{\prime \prime \prime}\right)= \pm\left|\sum_{i=1}^{n}(-1)^{i-1} L_{r+i} \omega^{(j-(1 / 2))(k-1)}\right|,
$$

for $j=1,2, \ldots,(n-1) / 2$, and

$$
\lambda_{(n+1) / 2}\left(A_{r, n}^{\prime \prime \prime}\right)=\sum_{i=1}^{n} L_{r+i}
$$

So

$$
\begin{aligned}
\left|\lambda_{j}\left(A_{r, n}^{\prime \prime \prime}\right)\right| & \leq \sum_{i=1}^{n}\left|(-1)^{i-1} L_{r+i}(-1)^{i-1}\right| \\
& =\sum_{i=1}^{n} L_{r+i}, \quad\left(j=1,2, \ldots, \frac{n+1}{2}\right) .
\end{aligned}
$$

By (66) and (67), we have

$$
\max _{0 \leq i \leq(n+1) / 2}\left|\lambda_{i}\left(A_{r, n}^{\prime \prime \prime}\right)\right|=\sum_{i=1}^{n} L_{r+i} .
$$

Since all skew left circulant matrices are symmetrical, by Lemma 9 and (12) and (68), we obtain

$$
\left\|A_{r, n}^{\prime \prime \prime}\right\|_{2}=L_{r+n+2}-L_{r+2} .
$$

Theorem 25. Let $A_{r, n}^{\prime \prime}=\operatorname{SLCirc}\left(L_{r+1}, \ldots, L_{r+n}\right)$ be skew left circulant matrix; the bounds for the spread of $A_{r, n}^{\prime \prime}$ are

$$
2 L_{r+n} \leq s\left(A_{r, n}^{\prime \prime}\right) \leq \begin{cases}\sqrt{M-\frac{2}{n} N^{2}}, & \text { if } n \text { isodd } \\ \sqrt{M}, & \text { if } n \text { is even }\end{cases}
$$

where

$$
\begin{gathered}
M=2 n\left(L_{r+n} L_{r+n+1}-L_{r+1} L_{r}\right), \\
N=L_{r+n-1}+L_{r-1} .
\end{gathered}
$$

Proof. Since $A_{r, n}^{\prime \prime}$ is a symmetric matrix, by (20),

$$
s\left(A_{r, n}^{\prime \prime}\right) \geq 2 \max _{i \neq j}\left|a_{i j}\right|=2 L_{r+n} .
$$

The trace of $A_{r, n}^{\prime \prime}$ is, if $n$ is odd,

$$
\begin{aligned}
\operatorname{tr} & \left(A_{r, n}^{\prime \prime}\right) \\
& =L_{r+1}-L_{r+2}+L_{r+3}-\cdots+L_{r+n} \\
& =L_{r+1}+L_{r+1}+L_{r+3}+\cdots+L_{r+n-2} \\
& =2 L_{r+1}+L_{r+1}+L_{r+2}+\cdots+L_{r+n-3} \\
& =2 L_{r+1}+\sum_{i=1}^{n-3} L_{r+i} .
\end{aligned}
$$

By (12), we have

$$
\operatorname{tr}\left(A_{r, n}^{\prime \prime}\right)=L_{r+n-1}+L_{r-1}=N
$$

Let $M=2 n\left(L_{r+n} L_{r+n+1}-L_{r+1} L_{r}\right)$; then, by (18), (62), and (74), we obtain

$$
s\left(A_{r, n}^{\prime \prime}\right) \leqslant \sqrt{M-\frac{2}{n} N^{2}} .
$$

If $n$ is even, then

$$
\begin{aligned}
\operatorname{tr}\left(A_{r, n}^{\prime \prime}\right)= & L_{r+1}-L_{r+1}+L_{r+3} \\
& -L_{r+3} \cdots-L_{r+n-1}=0 .
\end{aligned}
$$

By (18), (62), and (76), we have

$$
s\left(A_{r, n}^{\prime \prime}\right) \leqslant \sqrt{M}
$$

So the result follows. 


\section{Conclusion}

We discuss the invertibility of the skew circulant type matrices with any continuous Lucas numbers and present the determinant and the inverse matrices by constructing the transformation matrices. The four kinds of norms and bounds for the spread of these matrices are given, respectively. In [3], a new fast algorithm for optimal design of block digital filters (BDFs) is proposed based on skew circulant matrix. The reason why we focus our attention on skew circulant is to explore the application of skew circulant in the related field in medicine image, image encryption, and realtime tracking. On the basis of existing application situation [4], we conjecture that SVD decomposition of skew circulant matrix will play an important role in CT-perfusion imaging of human brain. On the basis method of [8] and ideas of [5], we will exploit real-time tracking with kernel matrix of skew circulant structure. A novel chaotic image encryption scheme based on the time-delay Lorenz system is presented in [6] with the description of circulant matrix. We will exploit chaotic image encryption algorithm based on skew circulant operation.

\section{Conflict of Interests}

The authors declare that there is no conflict of interests regarding the publication of this paper.

\section{Acknowledgments}

This research was supported by the Natural Science Foundation of Shandong Province (Grant no. ZR2011FL017), the National Nature Science Foundation of China (Grant no. F020701), and the AMEP of Linyi University, China.

\section{References}

[1] A. Daher, E. H. Baghious, and G. Burel, "Fast algorithm for optimal design of block digital filters based on circulant matrices," IEEE Signal Processing Letters, vol. 15, pp. 637-640, 2008.

[2] V. C. Liu and P. P. Vaidyanathan, "Circulant and skew-circulant matrices as new normal-form realization of IIR digital filters," IEEE Transactions on Circuits and Systems, vol. 35, no. 6, pp. 625-635, 1988.

[3] D. Q. Fu, Z. L. Jiang, Y. F. Cui, and S. T. Jhang, "A new fastalgorithm for optimal design of block digital filters by skewcyclic convolution," IET Signal Processing, p. 6, 2014.

[4] H.-J. Wittsack, A. M. Wohlschläger, E. K. Ritzl et al., "CTperfusion imaging of the human brain: advanced deconvolution analysis using circulant singular value decomposition," Computerized Medical Imaging and Graphics, vol. 32, no. 1, pp. 67-77, 2008.

[5] J. F. Henriques, R. Caseiro, P. Martins, and J. Batista, "Exploiting the circulant structure of tracking-by-detection with kernels," in Computer Vision-ECCV 2012, vol. 7575 of Lecture Notes in Computer Science, pp. 702-715, Springer, Berlin, Germany, 2012.

[6] X. Huang, G. Ye, and K.-W. Wong, "Chaotic image encryption algorithm based on circulant operation," Abstract and Applied Analysis, vol. 2013, Article ID 384067, 8 pages, 2013.
[7] Y. Jing and H. Jafarkhani, "Distributed differential space-time coding for wireless relay networks," IEEE Transactions on Communications, vol. 56, no. 7, pp. 1092-1100, 2008.

[8] M. J. Narasimha, "Linear convolution using skew-cyclic convolutions," IEEE Signal Processing Letters, vol. 14, no. 3, pp. 173-176, 2007.

[9] T. A. Gulliver and M. Harada, "New nonbinary self-dual codes," IEEE Transactions on Information Theory, vol. 54, no. 1, pp. 415417, 2008.

[10] P. J. Davis, Circulant Matrices, John Wiley \& Sons, New York, NY, USA, 1979.

[11] Z. L. Jiang and Z. X. Zhou, Circulant Matrices, Chengdu Technology University Publishing, Chengdu, China, 1999.

[12] D. Bertaccini and M. K. Ng, "Skew-circulant preconditioners for systems of LMF-based ODE codes," in Numerical Analysis and Its Applications, vol. 1988 of Lecture Notes in Computer Science, pp. 93-101, Springer, Berlin, Germany, 2001.

[13] R. H. Chan and X.-Q. Jin, "Circulant and skew-circulant preconditioners for skew-Hermitian type Toeplitz systems," BIT Numerical Mathematics, vol. 31, no. 4, pp. 632-646, 1991.

[14] R. H. Chan and K.-P. Ng, "Toeplitz preconditioners for Hermitian Toeplitz systems," Linear Algebra and Its Applications, vol. 190, pp. 181-208, 1993.

[15] T. Huckle, "Circulant and skew circulant matrices for solving Toeplitz matrix problems," SIAM Journal on Matrix Analysis and Applications, vol. 13, no. 3, pp. 767-777, 1992.

[16] J. N. Lyness and T. Sørevik, "Four-dimensional lattice rules generated by skew-circulant matrices," Mathematics of Computation, vol. 73, no. 245, pp. 279-295, 2004.

[17] H. Karner, J. Schneid, and C. W. Ueberhuber, "Spectral decomposition of real circulant matrices," Linear Algebra and Its Applications, vol. 367, pp. 301-311, 2003.

[18] J. Li, Z. L. Jiang, N. Shen, and J. W. Zhou, "On optimal backward perturbation analysis for the linear system with skew circulant coefficient matrix," Computational and Mathematical Methods in Medicine, vol. 2013, Article ID 707381, 7 pages, 2013.

[19] D. V. Jaiswal, “On determinants involving generalized Fibonacci numbers," The Fibonacci Quarterly, vol. 7, pp. 319-330, 1969.

[20] D. A. Lind, "A Fibonacci circulant," The Fibonacci Quarterly, vol. 8, no. 5, pp. 449-455, 1970.

[21] L. Dazheng, "Fibonacci-Lucas quasi-cyclic matrices," The Fibonacci Quarterly, vol. 40, no. 3, pp. 280-286, 2002.

[22] S.-Q. Shen, J.-M. Cen, and Y. Hao, "On the determinants and inverses of circulant matrices with Fibonacci and Lucas numbers," Applied Mathematics and Computation, vol. 217, no. 23, pp. 9790-9797, 2011.

[23] Y. Gao, Z. L. Jiang, and Y. P. Gong, "On the determinants and inverses of skew circulant and skew left circulant matrices with Fibonacci and Lucas numbers," WSEAS Transactions on Mathematics, vol. 12, no. 4, pp. 472-481, 2013.

[24] X. Y. Jiang, Y. Gao, and Z. L. Jiang, "Determinantsand inverses of skew and skew left circulant matrices involving the $k$ Fibonacci numbers in communications-I," Far East Journal of Mathematical Sciences, vol. 76, no. 1, pp. 123-137, 2013.

[25] X. Y. Jiang, Y. Gao, and Z. L. Jiang, "Determinants and inverses of skew and skew left circulant matrices involving the $k$ Lucas numbers in communications-II," Far East Journal of Mathematical Sciences, vol. 78, no. 1, pp. 1-17, 2013.

[26] S. Solak, "On the norms of circulant matrices with the Fibonacci and Lucas numbers," Applied Mathematics and Computation, vol. 160, no. 1, pp. 125-132, 2005. 
[27] A. İpek, "On the spectral norms of circulant matrices with classical Fibonacci and Lucas numbers entries," Applied Mathematics and Computation, vol. 217, no. 12, pp. 6011-6012, 2011.

[28] S. Shen and J. Cen, "On the bounds for the norms of $r$ circulant matrices with the Fibonacci and Lucas numbers," Applied Mathematics and Computation, vol. 216, no. 10, pp. 2891-2897, 2010.

[29] M. Akbulak and D. Bozkurt, "On the norms of Toeplitz matrices involving Fibonacci and Lucas numbers," Hacettepe Journal of Mathematics and Statistics, vol. 37, no. 2, pp. 89-95, 2008.

[30] A. Bose, R. S. Hazra, and K. Saha, "Spectral norm of circulanttype matrices," Journal of Theoretical Probability, vol. 24, no. 2, pp. 479-516, 2011.

[31] L. Mirsky, "The spread of a matrix," Mathematika, vol. 3, pp.127130, 1956.

[32] R. Sharma and R. Kumar, "Remark on upper bounds for the spread of a matrix," Linear Algebra and Its Applications, vol. 438, no. 11, pp. 4359-4362, 2013.

[33] L. Mirsky, "Inequalities for normal and Hermitian matrices," Duke Mathematical Journal, vol. 24, no. 4, pp. 591-599, 1957.

[34] E. R. Barnes and A. J. Hoffman, "Bounds for the spectrum of normal matrices," Linear Algebra and Its Applications, vol. 201, pp. 79-90, 1994.

[35] E. Jiang and X. Zhan, "Lower bounds for the spread of a Hermitian matrix," Linear Algebra and Its Applications, vol. 256, pp. 153-163, 1997.

[36] R. Bhatia and R. Sharma, "Some inequalities for positive linear maps," Linear Algebra and Its Applications, vol. 436, no. 6, pp. 1562-1571, 2012.

[37] J. Wu, P. Zhang, and W. Liao, "Upper bounds for the spread of a matrix," Linear Algebra and Its Applications, vol. 437, no. 11, pp. 2813-2822, 2012.

[38] C. R. Johnson, R. Kumar, and H. Wolkowicz, "Lower bounds for the spread of a matrix," Linear Algebra and Its Applications, vol. 71, pp. 161-173, 1985. 


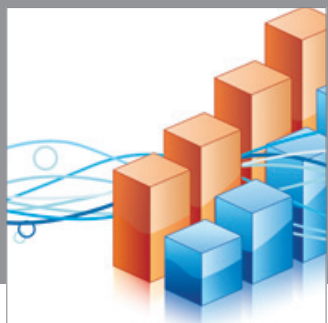

Advances in

Operations Research

mansans

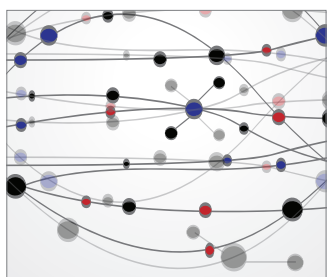

The Scientific World Journal
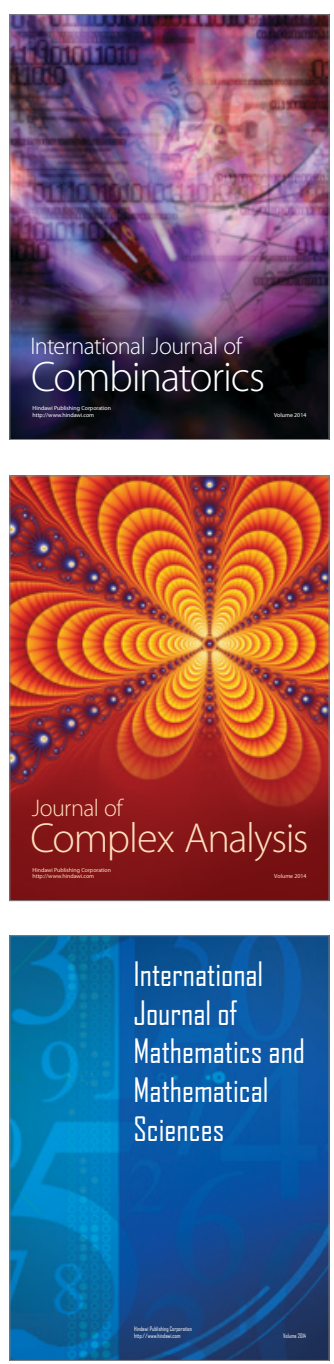
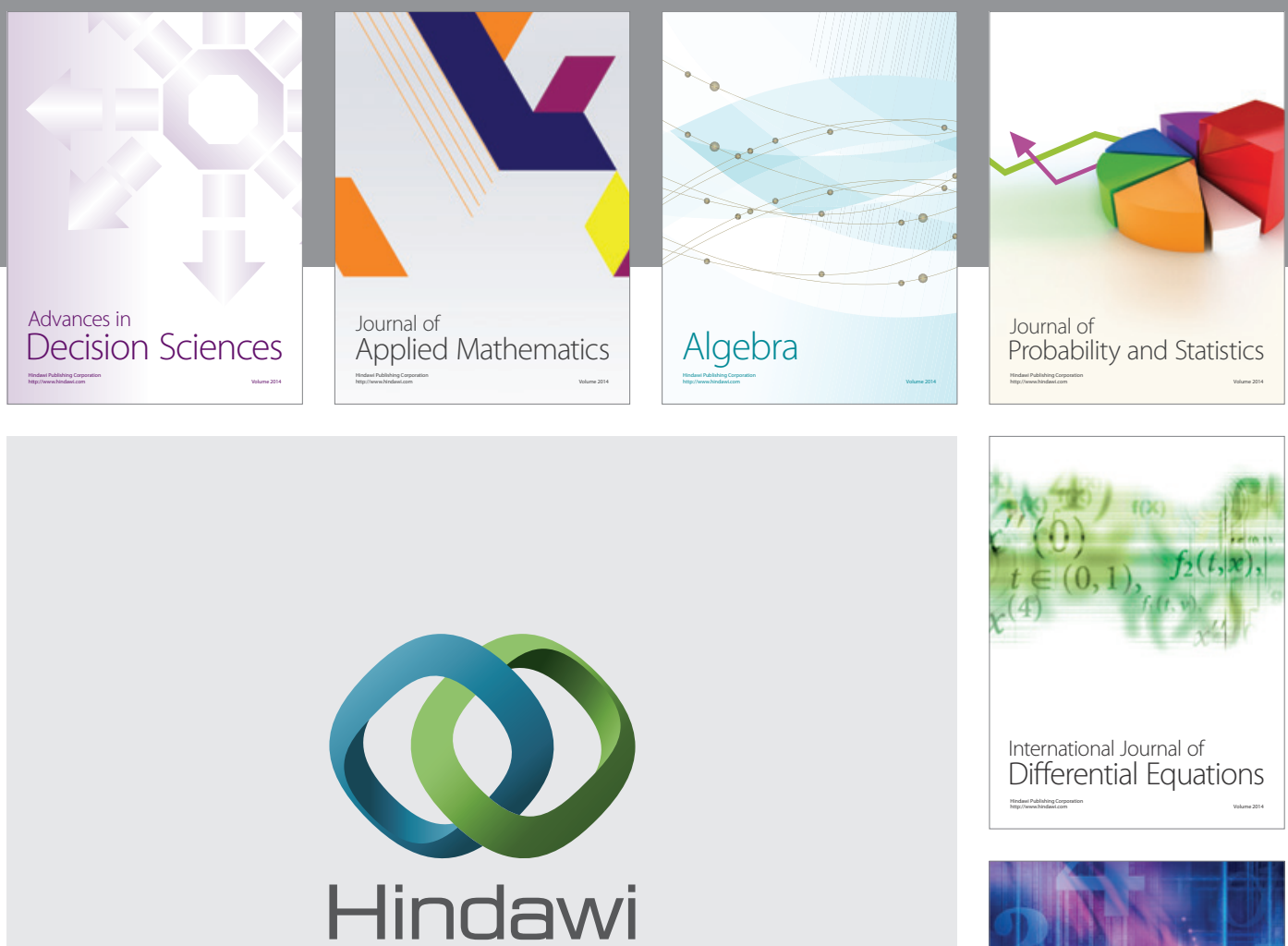

Submit your manuscripts at http://www.hindawi.com
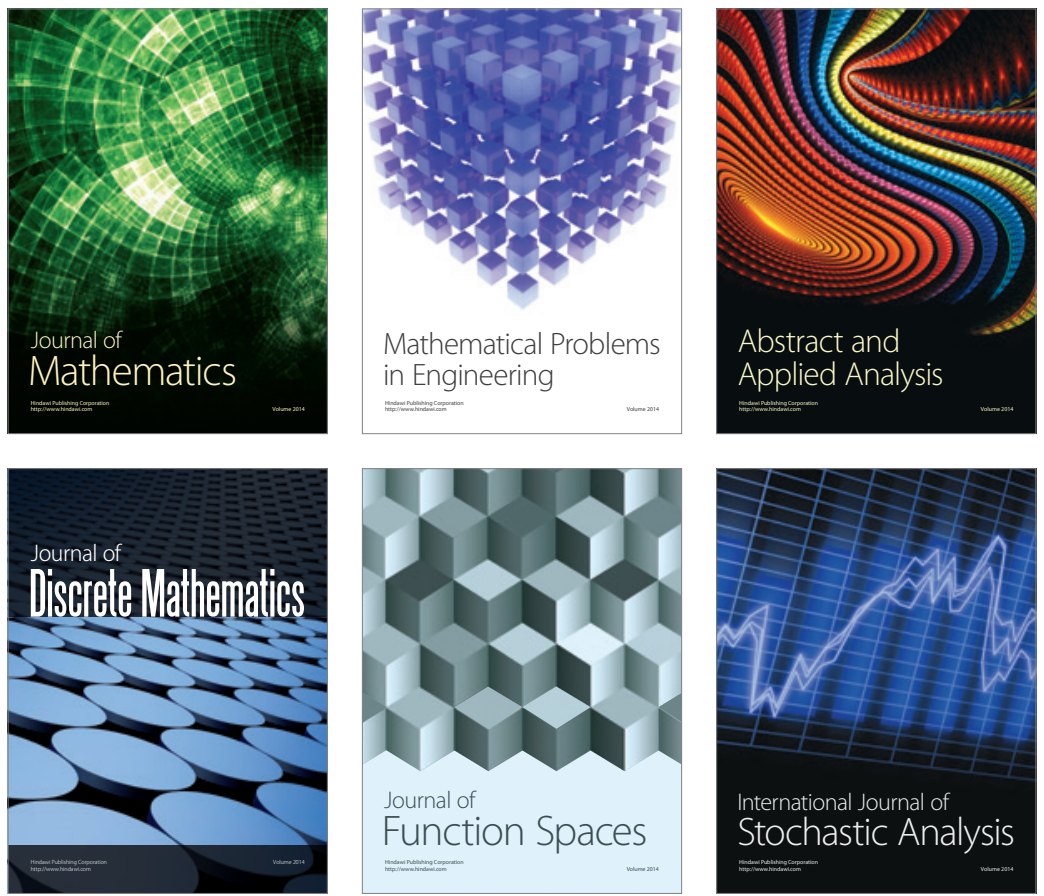

Journal of

Function Spaces

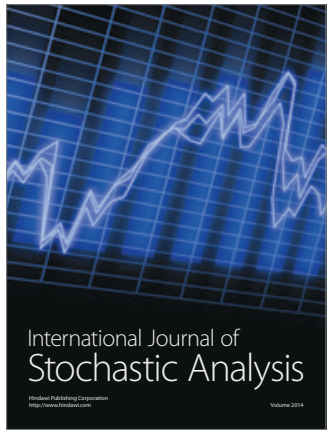

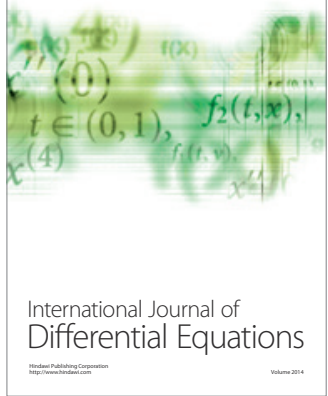
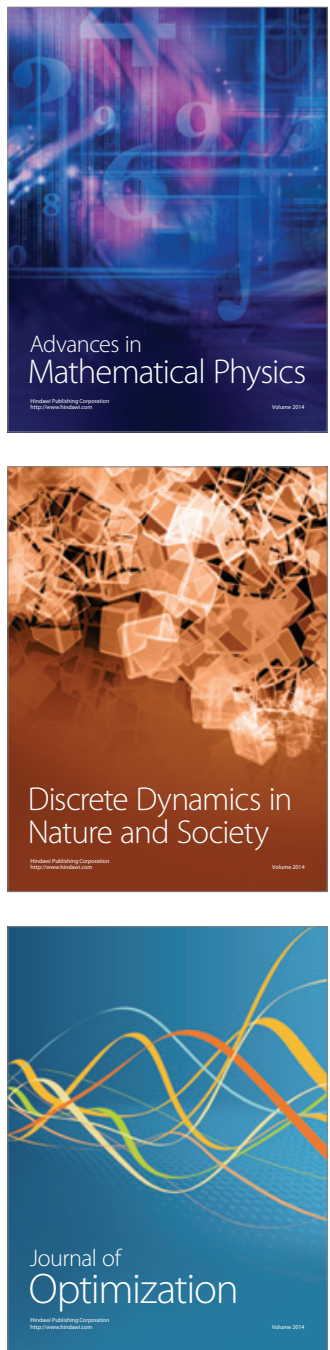